\title{
Synthetic Hydrogels Break Down by Gypsum and Fertilizers by Making These Unsuitable for Agricultural Uses
}

\author{
Virender Singh Lather* \\ Former Principal Scientist, ICAR-IARI Regional Station Karnal, India
}

*Corresponding author: Virender Singh Lather, Former Principal Scientist, ICAR-IARI Regional Station Karnal, India

\begin{abstract}
Hydrogels or super absorbents polymers (SAP) are used in agriculture for over 40 years and cross-linked polymers which absorb water 400 to 1500 times to dry weight. Hydrogels categorized into 3 classes: 1 . Naturally occurring polymers 2. Semi-synthetic polymers derived from cellulose by chemically combined with petrochemicals. 3. Synthetic polymers made from petrochemicals and polyacrylamides (PAM). The ICAR also accomplished a synthetic polymer 'Pusa hydrogel' to reduce the irrigation requirements of the crops. The absorptive capability of hydrogel is affected by its physical-chemical composition and environmental factors. Recent novel research findings that "Gypsum precipitates the gel of synthetic hydrogels into the fluid and make them unsuitable for agricultural uses" raised serious concerns and limitation of their use in agricultural for absorption and retention of water for delays onset of permanent wilting point in the crop production.
\end{abstract}

Keywords: Herbal hydrogel; Synthetic hydrogel; Pusa hydrogel; Gypsum; Polyacrylamides

\section{Introduction}

Hydrophilic gels or "hydrogels", which are commonly known as super absorbents polymers (SAP) have been used in agriculture for over 40 years and cross-linked polymers which absorb water 400 to 1500 times to their dry weight. Most of hydrogels were destined for non-agricultural diverse applications such as baby diapers, oil recovery, food processing, medical, personal care and hygiene. Hydrogels are categorized into three classes: 1 . Naturally occurring polymers are starch-based polysaccarides that made from grain crops such as corn, wheat and gums of various plants and commonly used in the food industry as thickening agents. 2. Semi-synthetic polymers derived from cellulose by chemically combined with petrochemicals. 3. Synthetic polymers made from petrochemicals and polyacrylamides (PAM) are one of the most popular polymers that chemically linked to prevent them from dissolving in solution and used for increase water holding capacity, erosion control, canal sealing, and water clarification. The absorptive capability of hydrogels is affected by their physical-chemical composition and environmental factors, such as the dissolved salts in water solution [1]. The ICAR-Indian Council of Agricultural Research also accomplished Pusa hydrogel(Semi-synthetic crosslinked hydrophilic polymer) for improving the water use efficiency of agricultural crops under limited irrigation and rainfed conditions with salient features as i)Cellulosic (a natural polymer) backbone, Ii) Free of the toxic monomer (acrylamide), iii) Absorbs water a minimum of 350 times of its dry weight and gradually releases it, iv) Reduces irrigation requirements of crops and delays onset of permanent wilting point(Anonymous,2018).However, even if companies producing SAP crystals are promoting their practicality and versatility, the scientific community is questioning about their real utility.

Linda Chalker-Scott [2] from Washington State University pointed out, since commonly used watering crystals are made out of non-renewable materials, whose monomers can be toxic (e.g. acrylamide), the potential risks of their usage are way higher than the benefits of water storage and controlled release that can be obtained in many other ways with lower environmental impact. 
Similar observations reported by Lather [3] and Lather et al. [4] that 'The Synthetic hydrogels have not become popular because environmental concerns as most of them contain polyvinyl alcohols or polyacrylamides which are considered carcinogenic and nonbiodegradable'. Further during refinement of water and resources conservation DSR-HHT (Direct Seeded Rice -Herbal Hydorogel Technology), the author who is now a ICAR-pensioner and working as a farmer observed an interesting phenomenon that 'The gels of synthetic hydrogels including Pusa hydrogel precipitated into liquid within few minutes in contact with Gypsum (CaSO4.2H2O calcium sulphate dehydrate), a soft sulphate mineral mines from dry land sandy soils and widely used as fertilizer and amendments for salt affected soils.

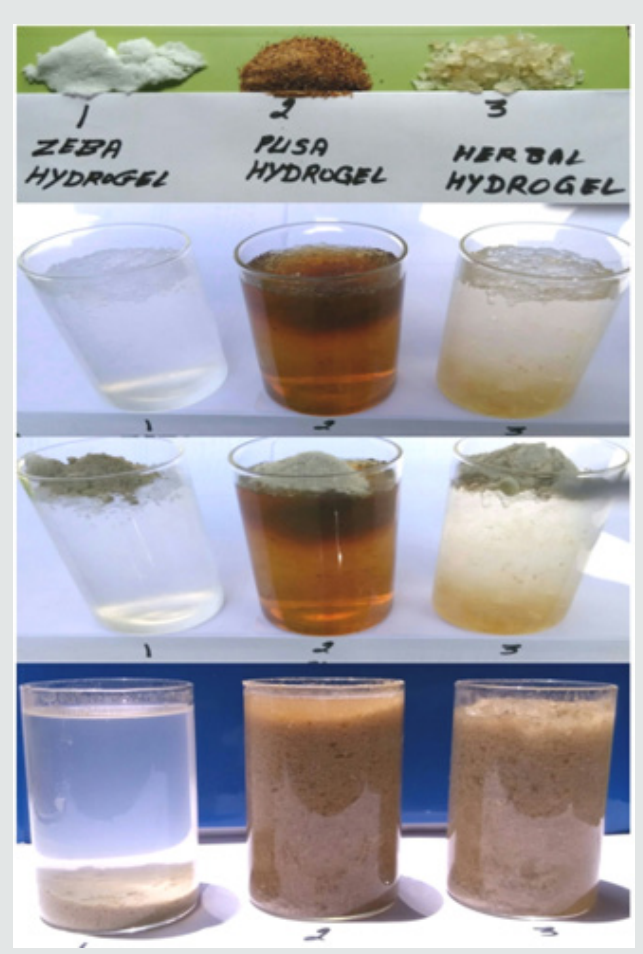

Figures 1: Precipitation of the gels of synthetic hydrogels in contact with the Gypsum.

These observations were validated further by conducting a simple experiment by taking three glass beakers, each filled with $150 \mathrm{ml}$ water and added two-gram synthetic hydrogels (Zeba \& Pusa hydrogel) and herbal hydrogel each in separate beaker. Then allows them absorbs the water for overnight to form it a nice gel and then added one-gram gypsum to each beaker, mixed it well. Zeba gels breakdown/precipitated into fluid within one minute, whereas gel of Pusa hydrogel precipitated in about 15 minutes, but gel of herbal hydrogel showed stability against gypsum (Figure 1). This research finding again raised the serious concerns on suitability of synthetic hydrogels for agricultural uses because chemical constituents of gypsum present in all the major soils of the world except hill zone where soils are generally acidic in nature. Similar findings reported by Meena [5] that 'No yield improvement was observed with hydrogel application' and Raza et al. [6] that 'Higher moisture can be retained in rooting media with application of soil additives (Qemisoyl, farm yard manure, gypsum and compost etc) as the sole application to achieve higher levels of soil water retention for the crop production in the vulnerable and drought prone rainfed ecology [7]. However, the combined use of these soil additives hydrogels- Qemisoyl with gypsum etc did not show any superiority over their sole application.

Therefore these recent novel research findings that 'Gypsum precipitates synthetic hydrogels and make them unsuitable for agricultural uses' raised serious concerns and confirmed the limitations of their use in agricultural sector because all major soils of the world possessed the constituents of gypsum particularly the sandy soils of rainfed ecology from where gypsum mines as the mineral and also pointed out the potential risks of their usage are way higher than the benefits of water absorption and retention that can be obtained with lower environmental impact in many other ways by using traditional soil additives such as FYM, gypsum, compost etc.

\section{References}

1. Landis TD, Haase DL (2012) Applications of Hydrogels in the Nursery and During Out-planting. USDA Forest Service Proceedings pp. 53-58.

2. Linda Chalker-Scott http://www.theinformedgardener.com is also.

3. Lather VS, Kumar A, Chopra NK, Choudhary D, Yadav RN (2015) 'Tragacanth-Katira Gel' and Farmer's Friendly Seed Priming-Hydrogel Coating Technology for Water Saving, Making Agriculture Sustainable and Resilient to Climatic Variability. 33(2): 1167-1171.

4. Lather (2016) Blog: Direct-Seeded Rice Can be a Success with Cheap Herbal Hydrogel-Coated Seeds.

5. Meena RP, Sharma RK, Tripathi SC, Gill SC, Chhokar RS, et al. (2015) Influence of hydrogel, irrigation and nutrient levels on wheat productivity. J Wheat Research7(2): 19-22.

6. Raza MSU, Ahmed ZIA, Malik MA, Ijaz SS (2015) Effective soil additives for improved soil water retention. Pak. J Agri Sci 52(2): 461-466.

7. (2018) Anonymous Accomplishments: Pusa Hydrogel for efficient water utilization. 
(c) (i)

This work is licensed under Creative Commons Attribution 4.0 License

To Submit Your Article Click Here:

Submit Article

DOI: 10.32474/CIACR.2018.05.000221

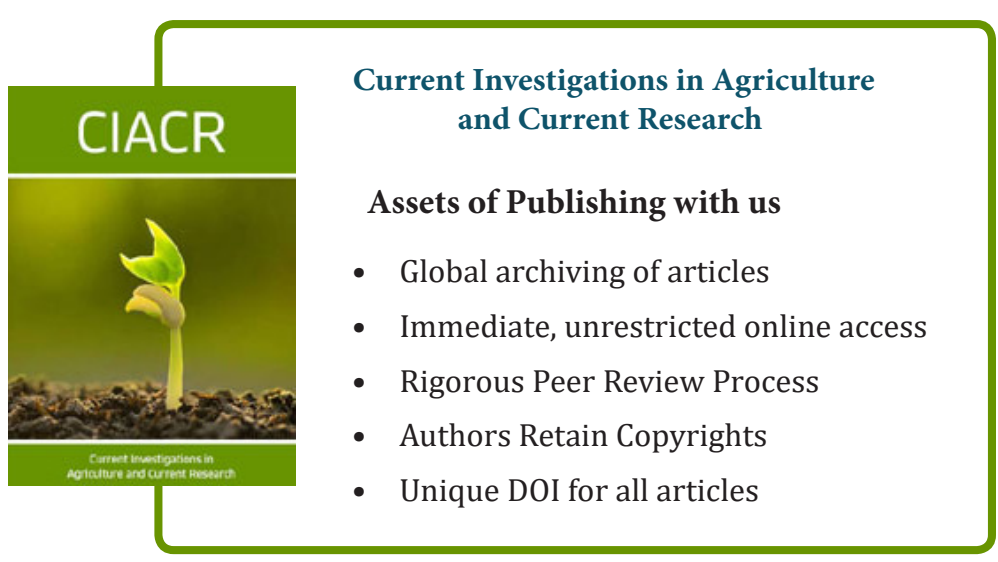

